\title{
NDF of the far zone field radiated by square sources
}

\author{
Ehsan Akbari Sekehravani*(1), Giovanni Leone (1), and Rocco Pierri ${ }^{(1)}$ \\ (1) Dipartimento di Ingegneria, Università della Campania "Luigi Vanvitelli", \\ Via Roma 29, Aversa, CE, Italy
}

\begin{abstract}
We consider the evaluation of the Number of Degrees of Freedom (NDF) of the field radiated by square sources in the far zone. The analysis is performed by employing a Singular-Value Decomposition (SVD) of the radiation operator in the two-dimensional scalar case. To this end, we start the analysis from simple geometries like two parallel strips and angle strips where analytical results can be established. For sufficiently spaced strip sources, the NDF depends only on their total electrical length. Then results for square sources follow on the same line. Finally, the numerical investigation of the case of concentric square sources, leading to an NDF independent on the inner source, opens the way to the discussion of the NDF of a full square source. In this case, it results that it depends only on the source perimeter.
\end{abstract}

\section{Introduction}

Determining radiated fields in the frequency domain for a known source embedded in a homogeneous background medium is defined as the radiation problem. In contrast, the inverse source problem defines reconstructing a current source from its radiated field knowledge.

The NDF in inverse problems is the maximum amount of information that can be recovered from the measured data in the presence of noise [1]. It describes the number of independent and significant parameters to reproduce the radiated field with a given degree of accuracy [2]. Moreover, it is relevant in the source imaging problem. In [2], the case of a strip current has been considered. However, the general 2D case is still an open problem, except for the circular geometry [3,4], even though this information is of great importance in solving inverse source problems.

The SVD provides a way to evaluate the NDF. In the following, the SVD is evaluated for each source shape, and the behavior of the singular values predicted when possible.

The outline of the paper is as follows. In section 2, we introduce the formulation of the problem and recall the analytical result for a single strip that is used throughout the paper. Next, section 3 presents the two parallel equal strips. In section 4 , we show the effectiveness of the angle between two strips on the NDF. Section 5 presents square sources with different configurations.

\section{Formulation of the problem}

This section aims to provide some mathematical preliminaries and notations used in the following sections. The two-dimensional geometries to be and examined consists of electric current sources embedded in a homogeneous medium with dielectric permittivity $\epsilon_{0}$ and magnetic permeability $\mu_{0}$.

We first recall the results of the NDF of the radiated field by an electric strip current $J$. The electric field is radiated by this source, which is assumed not to vary along the $Z$ direction and supported over the interval $I=[-a, a]$ of the $\mathrm{x}$-axis. The radiated field is collected in the far zone over the angular observation sector $[-\pi, \pi]$.

Now, let be $u=\cos \theta$, the relation between the electric current distribution $J(x)$, and the radiated electric field $E(u)$ is given in terms of the Fourier transform operator acting on functions of compact support by

$$
E(u)=\int_{-a}^{\mathrm{a}} J(\mathrm{x}) e^{j \beta u x} d x=\mathcal{L}_{u}(J(x))
$$

Since the eigendecomposition of $\mathcal{L}_{u}$ is known in terms of prolate spheroidal wave functions [5], estimating the NDF needs to compute the SVD of Equation (1). It has been shown that the eigenvalues are almost constant for $n<$ $[4 a / \lambda]$, where [.] stands for the nearest integer; after this index, they abruptly decay [6].

When we consider the $\theta$ angle as an observation variable, a different operator, say $\mathcal{L}_{\theta}$, must be considered and its SVD analyzed. However, it can be expected that, though the singular values are not the same for both operators $\mathcal{L}_{\theta}$ and $\mathcal{L}_{u}$, the NDF is the same, and the only difference is in shaping their behavior [3]. Hence, the estimated NDF are provided by

$$
N D F=\left[\frac{4 a}{\lambda}\right]
$$

\section{Two parallel equal strips}

The same analysis as the one strip is here extended to address two parallel equal stripes along the $\mathrm{x}$-axis is shown in Figure 1. They have no contact physically with each 
other. The two strips are located at $I_{1}=[-a, a]$ and $I_{2}=$ $[-a, a]$, respectively, and $d=2 b$ is the distance between them. The goal is to investigate the relationship between the distance between two strips and the NDF, furthermore illustrating a rule that the whole NDF of two strips can compute by summing the NDF of each strip.

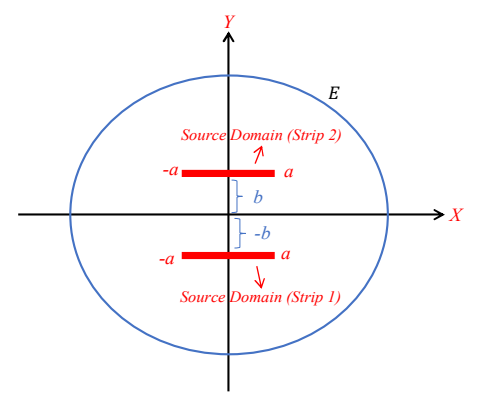

Figure 1. The geometry of the problem

Firstly, the radiated far field for strips one and two can define by Equations (3) and (4), respectively.

$$
\begin{gathered}
E_{1}(\theta)=\int_{-a}^{a} J_{1}\left(x_{1}\right) e^{j \beta\left[x_{1} \cos \theta-b \sin \theta\right]} d x_{1}= \\
\mathcal{T}_{1}\left(J_{1}\left(x_{1}\right)\right)=e^{-\mathrm{j} \beta b \sin \theta} \mathcal{L}_{1}\left(J_{1}\left(x_{1}\right)\right)
\end{gathered}
$$

Where $\mathcal{T}_{1}\left(J_{1}\left(x_{1}\right)\right)$ is a linear operator and $\mathcal{L}_{1}\left(J_{1}\left(x_{1}\right)\right)$ is

and

$$
\mathcal{L}_{1}\left(J_{1}\left(x_{1}\right)\right)=\int_{-\mathrm{a}}^{\mathrm{a}} J_{1}\left(x_{1}\right) e^{j \beta x_{1} \cos \theta} d x_{1}
$$

$$
\begin{gathered}
E_{2}(\theta)=\int_{-a}^{a} J_{2}\left(x_{2}\right) e^{j \beta\left[x_{2} \cos \theta+b \sin \theta\right]} d x_{2}= \\
\mathcal{T}_{2}\left(J_{2}\left(x_{2}\right)\right)=e^{j \beta b \sin \theta} \mathcal{L}_{2}\left(J_{2}\left(x_{2}\right)\right)
\end{gathered}
$$

Where $\mathcal{T}_{2}\left(J_{2}\left(x_{2}\right)\right)$ is a linear operator and $\mathcal{L}_{2}\left(J_{2}\left(x_{2}\right)\right)$ is

$$
\mathcal{L}_{2}\left(J_{2}\left(x_{2}\right)\right)=\int_{-\mathrm{a}}^{\mathrm{a}} J_{2}\left(x_{2}\right) e^{j \beta x_{2} \cos \theta} d \mathrm{x}
$$

The relevant operator to be considered can be written as

$$
E(\theta)=\left[\begin{array}{llll}
e^{-j \beta b \sin \theta} & \mathcal{L}_{1} & e^{j \beta b \sin \theta} & \mathcal{L}_{2}
\end{array}\right]\left[\begin{array}{l}
J_{1}\left(x_{1}\right) \\
J_{2}\left(x_{2}\right)
\end{array}\right]
$$

The adjoint operator of (3) and (4) are given by

$$
\mathcal{T}_{n}^{+}=\left\{\begin{array}{l}
\mathcal{T}_{1}^{+}=\mathcal{L}_{1}^{+}\left(E_{1}\right) e^{j \beta b \sin \theta} \\
\mathcal{T}_{2}^{+}=\mathcal{L}_{2}^{+}\left(E_{2}\right) e^{-j \beta b \sin \theta}
\end{array}\right.
$$

Where $\mathcal{L}_{n}^{+}$is the adjoint of the operator $\mathcal{L}_{n}$, and $n=1,2$

$$
\mathcal{L}_{n}^{+}\left(E_{n}\right)=\int_{-\pi}^{\pi} E_{n}(\theta) e^{-j \beta x_{n}^{\prime} \cos \theta} d \theta
$$

Now, Equation (8) reads as

$$
\mathcal{T}_{\mathrm{n}}^{+}=\left\{\begin{array}{l}
\mathcal{T}_{1}^{+}=\int_{-\pi}^{\pi} \mathrm{E}_{1}(\theta) \mathrm{e}^{-\mathrm{j} \beta x_{1}^{\prime} \cos \theta} \mathrm{e}^{\mathrm{j} \beta \mathrm{b} \sin \theta} \mathrm{d} \theta \\
\mathcal{T}_{2}^{+}=\int_{-\pi}^{\pi} \mathrm{E}_{2}(\theta) \mathrm{e}^{-\mathrm{j} \beta x_{2}^{\prime} \cos \theta} \mathrm{e}^{-\mathrm{j} \beta \mathrm{b} \sin \theta} \mathrm{d} \theta
\end{array}\right.
$$

For the NDF estimation, we can equivalently consider the operator [7]

$$
\left(\mathcal{T}^{+} \mathcal{T}\right)(J)=\left[\begin{array}{ll}
\mathcal{T}_{1}^{+} \mathcal{T}_{1} & \mathcal{T}_{1}^{+} \mathcal{T}_{2} \\
\mathcal{T}_{2}^{+} \mathcal{T}_{1} & \mathcal{T}_{2}^{+} \mathcal{T}_{2}
\end{array}\right]\left[\begin{array}{l}
J_{1}\left(x_{1}\right) \\
J_{2}\left(x_{2}\right)
\end{array}\right]
$$

Finally, Equation (11) reads as

$$
\left(\mathcal{T}^{+} \mathcal{T}\right)(J)=\left[\begin{array}{lll}
\mathcal{T}_{1}^{+} \mathcal{T}_{1} & \mathcal{L}_{1}^{+} e^{2 j \beta b \sin \theta} \mathcal{L}_{2} \\
\mathcal{L}_{1}^{+} e^{-2 j \beta b \sin \theta} \mathcal{L}_{2} & \mathcal{T}_{2}^{+} \mathcal{T}_{2}
\end{array}\right]\left[\begin{array}{l}
J_{1} \\
J_{2}
\end{array}\right]
$$

The kernel of the integral operator $\left(\mathcal{T}^{+} \mathcal{T}\right)(J)$ is provided by the Bessel function of the first kind and zeroth order. When $d$ is sufficiently large, say more than $0.25 \lambda$ (see Figure 2), the kernel norms and, consequently, the operator norms, are expected larger for the diagonal contributions than for the off-diagonal ones, so it results [7]

$$
\left(\mathcal{J}^{+} \mathcal{T}\right)(J) \cong\left[\begin{array}{lr}
\mathcal{L}_{1}^{+} \mathcal{L}_{1} & 0 \\
0 & \mathcal{L}_{2}^{+} \mathcal{L}_{2}
\end{array}\right]\left[\begin{array}{l}
J_{1}\left(x_{1}\right) \\
J_{2}\left(x_{2}\right)
\end{array}\right]
$$

Thus, denoting as $N D F_{1}$ and $N D F_{2}$ the NDF of the fields radiated by each strip, respectively, we find that the total NDF can be provided approximately by summing $N D F_{1}$ and $N D F_{2}$. The NDF of the source configuration can be given by

$$
N D F_{T} \approx N D F_{1}+N D F_{2}
$$

Since the eigenvalues of a diagonal block operator are the combination of each term, this result implies that the whole functional space of the radiated far fields can be approximately decomposed under two individual orthogonal subspaces. Figure 2 confirms the estimation Equation (14) numerically.

The same result can be extended to the case of an arbitrary number $M$ of radiated fields, so that Equation (14) reads as [7]

$$
N D F_{T} \approx \sum_{n=1}^{M} N D F_{n}
$$

Where $N D F_{T}$ stands for the NDF of the whole set of sources and $N D F_{n}$ is the NDF of each source.

Figure 2 illustrates the behavior of normalized singular values of the relevant operator (12) at varying distances $d$ between two strips. This comparison aims at finding the influence of distance $d$ on the NDF so to determine what its minimum value able to achieve the whole NDF. It results that for distances larger than $0.25 \lambda$, the behavior of the singular values does not change significantly, whereas 
after $0.25 \lambda$ it does. It can be concluded that the minimum distance is significant to achieve the whole NDF.

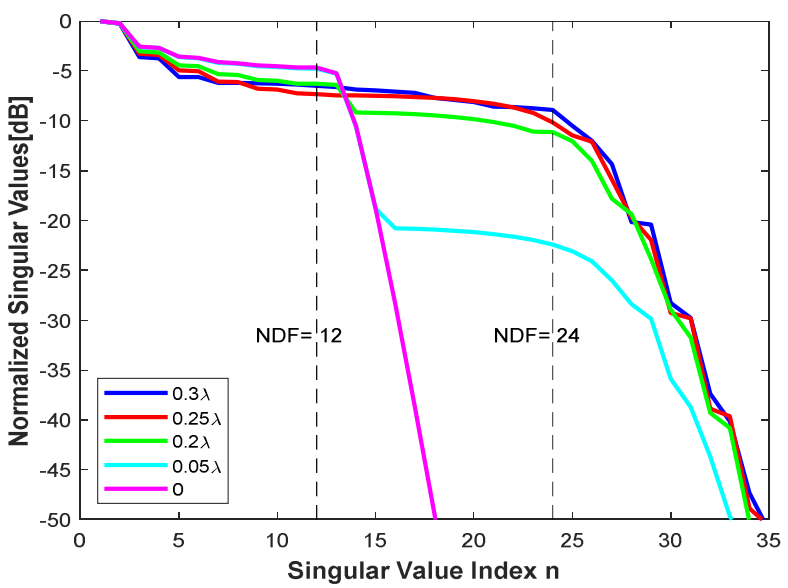

Figure 2. The normalized singular values of the relevant operator (12), with $a=3 \lambda$

\section{Angle strips}

We consider the geometry, as shown in Figure 3. By analyzing this geometry, we can investigate the influence of the angle between the two strips on the NDF. The angle between them is denoted by $\phi$. By the same analytical approach of section 3 , we can conveniently compute the whole NDF by summing the NDF of two strips.

We intend to compare varying angles between two strips to understand and find a minimum angle to achieve the whole NDF. Here, we have made some comparisons for different lengths of strip, considering one of them as an example. Figure 4 shows the behavior of normalized singular values of the relevant operator at different angles. The length of each strip is equal to $a=6 \lambda$. As can be seen, for angles larger than $\theta=3^{\circ}$, the behavior of singular values does not change significantly. However, for smaller angles, the behavior of singular values varies quickly; it appears two step-like behavior, and the number of singular values decreases. Consequently, the angle of the two strips is significant to achieve the whole NDF.

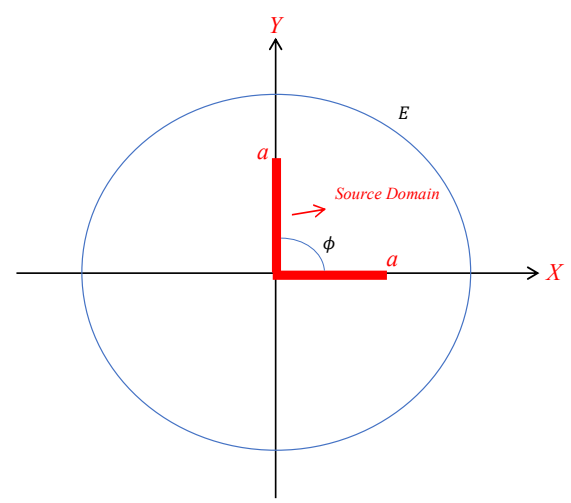

Figure 3. The geometry of the problem

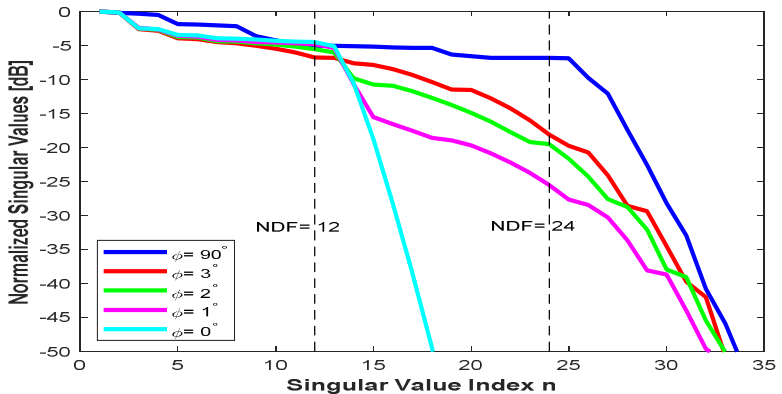

Figure 4. The normalized singular values of the relevant operator

\section{Square sources}

The purpose of this section is to apply the results of the previous ones to square sources and provide numerical results about NDF for three geometries. From the results of the previous Sections, it is expected that the overall electrical length of the source will be significant to achieve the whole NDF; in addition, it is observed that the source boundary may get the full NDF of a full square. To this end, one square source will be analyzed in subsection 5.1. Then, we will extend it to two concentric squares in subsection 5.2. In the end, subsection 5.3 will provide the full square case.

\subsection{Four sides of a square}

Let consider a square source that consists of four strips; the length of each strip is $2 a=12 \lambda$, as shown in Figure 5. As can be observed in Figure 6, the total NDF is equal to the summation of the NDF of the four strips, which can be obtained by (15). Consequently, the total electrical length of the square is essential to achieve the whole NDF.

\subsection{Two concentric squares}

We intend to address now the two square case. Figure 5 exhibits the geometry of two squares. The main goal is to examine how adding an inner square inside the outer square can affect the NDF. The question is that the internal square may be useless to achieve the NDF or not. We mean to examine whether the total NDF of two squares will be equal to the NDF of the outer square or not. To this end, we present a numerical simulation with different sizes of the inner square.

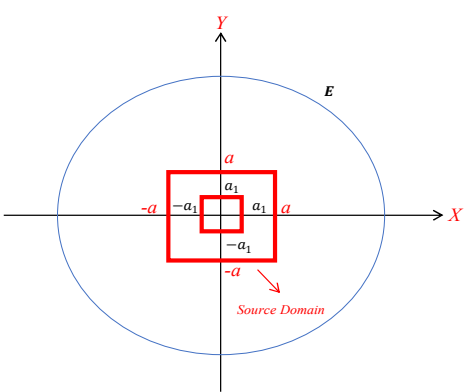

Figure 5. The geometry of the square sources 
As shown in Figure 6, changing the size of the inner square can affect the behavior of singular values only slightly; however, the NDF does not change since the change of the slope of the singular values curve occurs nearly always at the same index.

As can be observed, increasing the size of the inner square does not significantly change the behavior of singular values, and whatever the inner square be small, the behavior of singular values of the outer square will be close to the behavior of two squares. It can be concluded that the contribution of the inner square is negligible, and it is possible to ignore it to achieve the whole NDF. It means that the inner square source cannot affect the NDF, and it can affect only the behavior of singular values. Now a question arises how we can reconstruct the inner square source. It is challenging to know that the inner square is present or absent; thus, it needs more priory information. In this paper, we do not consider it.

\subsection{Full square}

This section intends to compare the full square to previous results obtained in subsection 5.2. The purpose is to illustrate the difference between these two geometries to obtain the NDF. Thus, it needs to introduce the relevant equation of the full square. Let us consider a 2D scalar current source $J(x, y)$ defined on a square shape. At a single frequency, the electric field $E(\theta)$ radiated in the far zone is provided by the linear integral operator

$$
\begin{gathered}
E(\theta)=\int_{-a}^{a} \int_{-a}^{a} J(x, y) e^{j \beta[x \cos \theta+y \sin \theta]} d x d y \\
=\mathcal{L}_{\theta}(J(x, y))
\end{gathered}
$$

For a numerical evaluation of the singular values of (16), we consider the case of a $12 \lambda \times 12 \lambda$ square source. The SVD of (16) is numerically computed by adequate discretization of both source and observation domains leading to a matrix. It can be seen in Figure 6 that the only difference between the two geometries is the behavior of the singular values before their exponential decay; furthermore, considering the full square does not increase the NDF. To conclude, the NDF of both geometries is the same, and in the 2D geometries, the source boundary is significant to obtain the NDF, which means the NDF depends on the boundary of the source, not the area.

A simple explanation of this result can be derived by resorting to the equivalence theorem. In fact, the same far field can be achieved either by the original square current source by (16) or by equivalent currents on its boundary. Accordingly, the corresponding subspaces must all have the same dimension, i.e., the same NDF.

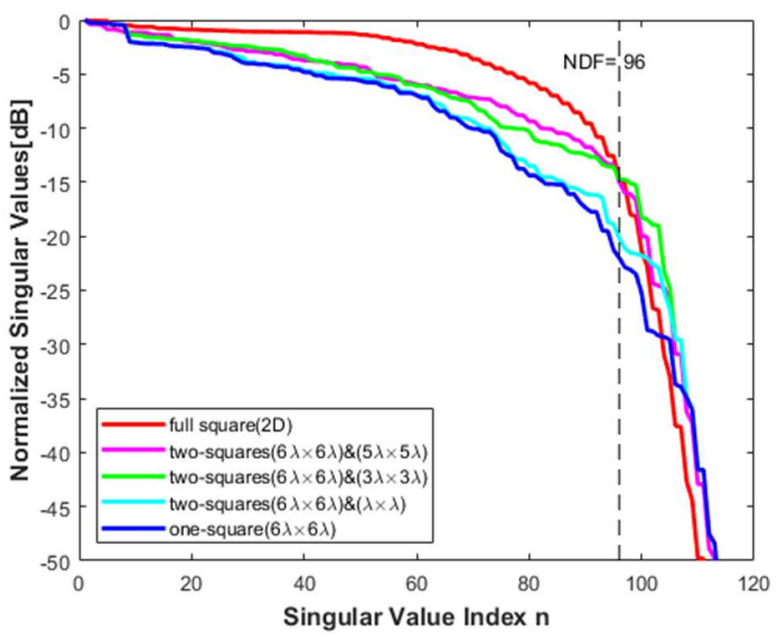

Figure 6. The normalized singular values of the relevant operators

\section{References}

[1] R. Piestun, and D. A. B. Miller, "Electromagnetic degrees of freedom of an optical system," Journal of the Optical Society of America A, vol. 17, no. 5, pp. 892-902, 2000/05/01, 2000.

[2] R. Solimene, M. A. Maisto, and R. Pierri, "Inverse source in the near field: the case of a strip current," Journal of the Optical Society of America A, vol. 35, no. 5, pp. 755-763, 2018/05/01, 2018.

[3] G. Leone, M. A. Maisto, and R. Pierri, "Inverse Source of Circumference Geometries: Svd Investigation Based on Fourier Analysis," Progress In Electromagnetics Research M, vol. 76, pp. 217-230, 2018.

[4] G. Leone, M. A. Maisto, and R. Pierri, "Application of Inverse Source Reconstruction to Conformal Antennas Synthesis," IEEE Transactions on Antennas and Propagation, vol. 66, no. 3, pp. 1436-1445, 2018.

[5] D. Slepian, and H. O. Pollak, "Prolate spheroidal wave functions, fourier analysis and uncertainty — I," The Bell System Technical Journal, vol. 40, no. 1, pp. 43-63, 1961.

[6] H. J. Landau, and H. Widom, "Eigenvalue distribution of time and frequency limiting," Journal of Mathematical Analysis and Applications, vol. 77, no. 2, pp. 469-481, 1980/10/01/, 1980.

[7] G. Leone, F. Munno, and R. Pierri, "Synthesis of Angle Arrays by the NDF of the Radiation Integral," accepted on IEEE Transactions on Antennas \& Propagation, 2020. 